\title{
Far field estimation using current distribution reconstructed by hemispherical near field
}

\author{
Yuzo Hayashi ${ }^{1 a)}$, Hiroyuki Arai ${ }^{1}{ }^{1}$, Shinya Iwanaga ${ }^{2}$, \\ and Satoshi Hori ${ }^{2}$ \\ ${ }^{1}$ Graduate School of Engineering, Yokohama National University, \\ 79-1 Tokiwadai, Hodogayaku, Yokohama, Kanagawa, Japan \\ ${ }^{2}$ KOJIMA INDUSTRIES CORPORATION, \\ Kurozasa Technical Center, Aichi, Japan
}

a)hayashi-yuzo-xb@ynu.jp

\begin{abstract}
Vehicular antenna are seriously affected by their body due to current flowing on the surface. Therefore, several near field measurements have been proposed to estimate the current distribution on the conductor surface by hemispherical scanning, but errors are not enough small by complex model in high frequency. This paper presents Hermitian transpose to improve the estimation, which is verified in simulations.
\end{abstract}

Keywords: far field estimation, current distribution, inverse problem, near field

Classification: Antennas and Propagation

\section{References}

[1] K. Nishikawa and Y. Asano, "Vertical radiation patterns of mobile antenna in UHF band," IEEE Trans. Veh. Technol., vol. 35, no. 2, May 1986. DOI:10.1109/ T-VT.1986.24071

[2] T. Basikolo, H. Arai, S. Hori, and S. Iwanaga, "Reconstruction of far field radiation pattern from hemispherical near field measurements," IEEE iWem. Pos1.47, Japan, 2018. DOI:10.1109/iWEM.2018.8536677

[3] K. Mei, "On the integral equations of thin wire antennas," IEEE Trans. Antennas Propag., vol. 13, no. 3, pp. 374-378, May 1965. DOI:10.1109/TAP.1965. 1138432

[4] M. R. Hestenes and E. Stiefel, "Methods of conjugate gradients for solving linear systems," J. Res. Natl. Bur. Stand., vol. 49, no. 6, p. 409, Dec. 1952. DOI:10.6028/jres.049.044

[5] FEKO, EM Software and Systems (www.feko.info).

[6] EEM-MOM, EEM (http://www.e-em.co.jp). 


\section{Introduction}

When an antenna is connected to a conductor, the entire conductor operates as an antenna due to the current flowing on the conductor surface. Therefore, its radiation pattern is largely different from the antenna element itself [1]. Vehicular antennas are necessary to measure the patterns including the effect of body. Far field measurement requires long distance because of large body size compared with wavelength. Conventional far field measurement methods, such as open site and large anechoic chamber, has a problem of high cost based on the size and space. Near field measurement, such as defocusing method, compact range, and extrapolation method, has the problem that it cannot be evaluated assuming real ground in the actual operating environment. A method of estimating the current distribution flowing on the conductor surface was proposed by the inverse problem from hemispherical near field measurement [2]. In this method, the electromagnetic field generated from the wave source is represented by an integral equation, and the current distribution is estimated by solving a simultaneous linear equation obtained by discretizing the conductor surface. Once the current distribution is obtained, the radiation pattern on real ground is estimated by using the reflection coefficient method. However, the method in [2] has large errors in high frequency. This paper presents the calculation algorithm to improve the accuracy by introducing Hermitian transpose into matrix formulation.

\section{Theory of current distribution estimation}

The current distribution on the conductor surface is estimated using the near field measured in the anechoic chamber. The electromagnetic field generated at the observation point $r^{\prime}$ by the current distribution $J$ of the conductor surface in the region $v$ at the position $r$ by the following equation,

$$
\begin{aligned}
\boldsymbol{E}\left(\boldsymbol{r}^{\prime}\right) & =-\frac{j \eta}{k} \int_{v}\left\{k^{2} \boldsymbol{J}(\boldsymbol{r})+\nabla \nabla \cdot \boldsymbol{J}(\boldsymbol{r})\right\} G\left(\boldsymbol{r}, \boldsymbol{r}^{\prime}\right) d \boldsymbol{v} \\
G\left(\boldsymbol{r}, \boldsymbol{r}^{\prime}\right) & =\frac{e^{-j k\left|\boldsymbol{r}-\boldsymbol{r}^{\prime}\right|}}{4 \pi\left|\boldsymbol{r}-\boldsymbol{r}^{\prime}\right|}
\end{aligned}
$$

where $\eta$ is the impedance and $k$ is the wave number in free space. In the calculation of the current distribution, the conductor surface is represented by linear elements based on the discretization with the moment method as shown in Fig. 1(b) [3], and the electric field generated by the current $J_{n}$ flowing in each linear element at any observation point $\boldsymbol{r}_{p}$ in the $p$ component $(p=x, y, z)$ of the orthogonal coordinate is expressed by the following equation,

$$
\begin{gathered}
\boldsymbol{E}_{p}(\boldsymbol{r})=\frac{j k \eta}{4 \pi} \sum_{n=1}^{N} J_{n}\left\{-\frac{1+j k R_{n}^{+}}{\left(k R_{n}^{+}\right)^{2}} \frac{\boldsymbol{r}_{p}-\boldsymbol{r}_{n p}^{+}}{R_{n}^{+}} e^{-j k R_{n}^{+}}\right. \\
\left.+\frac{1+j k R_{n}^{-}}{\left(k R_{n}^{-}\right)^{2}} \frac{\boldsymbol{r}_{p}-\boldsymbol{r}_{n p}^{-}}{R_{n}^{-}} e^{-j k R_{n}^{-}}-\frac{\Delta l_{n}}{R_{n}}\left(\hat{\boldsymbol{p}} \cdot \hat{\boldsymbol{l}_{n}}\right) e^{-j k R_{n}}\right\} \\
\left(R_{n}^{ \pm}=\left|\boldsymbol{r}_{p}-\boldsymbol{r}_{n p}^{ \pm}\right|, \boldsymbol{r}_{n p}^{ \pm}=\left(r_{n x}^{ \pm}, r_{n y}^{ \pm}, r_{n z}^{ \pm}\right), \boldsymbol{r}_{n p}=\left(r_{n x}, r_{n y}, r_{n z}\right), \boldsymbol{r}_{p}=\left(r_{x}, r_{y}, r_{z}\right)\right)
\end{gathered}
$$


As shown in Fig. 1(c), the center position of linear element $\mathrm{n}$ is represented by $\boldsymbol{r}_{n p}$, and $\boldsymbol{r}_{n p}^{+}$and $\boldsymbol{r}_{n p}^{-}$indicate the positions of both ends. Equation (3) can be expressed by matrix expression as in the following equation,

$$
[E]=[A][J]
$$

where $[\boldsymbol{E}]$ is near electric field distribution vector, $[\boldsymbol{A}]$ is coefficient matrix representing the radiation from each element determined by the model shape, and $[\boldsymbol{J}]$ is a current vector of each element. The current distribution $[\boldsymbol{J}]$ is calculated by solving the inverse problem of Eq. (4), however the inverse matrix cannot be calculated because $[A]$ is not regular. In [2], pseudo-inverse matrix is used for the underdetermined problem, and it gives not the least squares solution of Eq. (4), but the solution of minimum norm of $[\boldsymbol{J}]$. To estimate far field accurately, it is necessary to reconstruct current distribution $[J]$ as a least square solution of Eq. (4). To overcome this difficulty, in this paper, the Hermite transpose matrix of $[A]$ is applied to both sides of Eq. (4) as in the following equation,

$$
[\boldsymbol{A}]^{*}[\boldsymbol{E}]=[\boldsymbol{A}]^{*}[\boldsymbol{A}][\boldsymbol{J}]
$$

Equation (5) is a normal equation, and the solution of this equation is the least squares solution of Eq. (4). To calculate Eq. (5), this paper adopts conjugate gradient method, and iterate until the relative residuals on both sides decrease sufficiently [4].

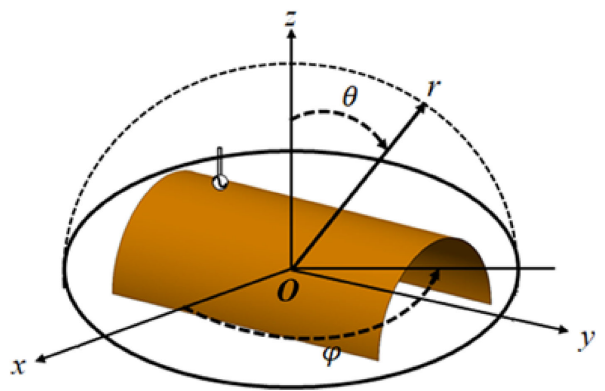

(a) Semi-cylindrical model

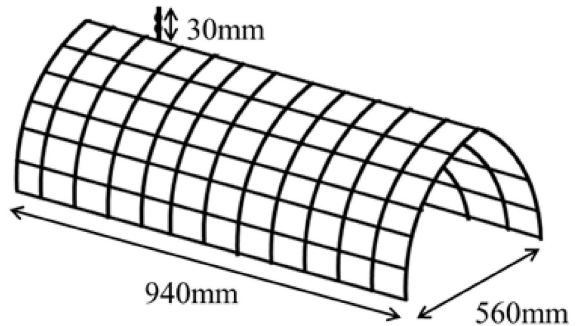

(b) Linear approximation

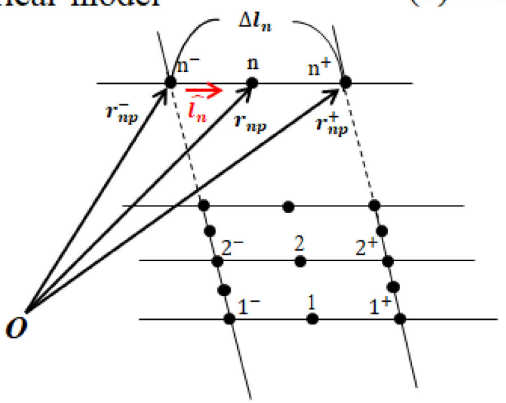

(c) Linear element

Fig. 1. Simulation model

\section{Simulation result}

In the beginning, we consider the model shown in Fig. 1(a). In addition, estimation is performed using the hemispherical near electric field in free space obtained by electromagnetic field analysis software FEKO [5], and the reference of far field in 
free space is also given by the same simulator. The near field is sampled at intervals of 5 degree in both the $\theta$ and $\phi$ directions. Linear approximation model which is shown in Fig. 1(b) is made by EEM-MOM [6], and coordinates of each linear element output from EEM-MOM are used for inverse problem calculation. Root mean square (RMS) is calculated by estimated far field and reference value. Fig. 2(a) shows the change in RMS for relative residuals of Eq. (5) at $75 \mathrm{MHz}$, $300 \mathrm{MHz}$ and $1.5 \mathrm{GHz}$. The estimation condition of the near field measurement radius is $1 \mathrm{~m}$, and the length of each element in the linear approximation model is $0.1 \lambda$ or less. The relative residual in the calculation of the inverse problem decreases in respective frequency, and the RMS converges sufficiently by reducing the relative residual to $10^{-6}$ or less. Fig. 2(b) shows far field estimation comparison of proposed method and method in [2] in yz plane. Proposed method can estimate far field more accurate than method in [2]. Next, we clarify the required conditions for the estimation. Fig. 2(c) shows the change in RMS for the measurement radius at each frequency. Since the minimum measuring radius is $0.6 \mathrm{~m}$ by the size of the model, the minimum measuring radius for wavelength is different at each frequency. The RMS becomes sufficiently small when the measurement radius is 1 to $2 \lambda$ or more. However, at $75 \mathrm{MHz}$, the measurement minimum radius is $0.15 \lambda$, which is the smallest of the measured radius with regards to wavelength, but the RMS is about $1 \%$. The estimation error is relatively small even when the measurement radius is small. Fig. 2(d) shows the change in RMS for the length of each element of the linear approximation model at each frequency. They are divided at least by 10 degree in the circumferential direction. The RMS is deteriorated as the linear element length increases at each frequency. At $75 \mathrm{MHz}$, the model size is small relative to the wavelength, therefore, the range within which changes is small, while the linear element is $0.2 \lambda$ or less, the RMS is $1 \%$ or less, and high accuracy estimation is obtained.

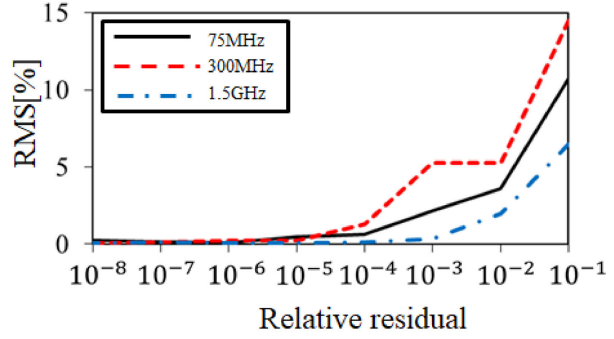

(a) RMS for relative residuals

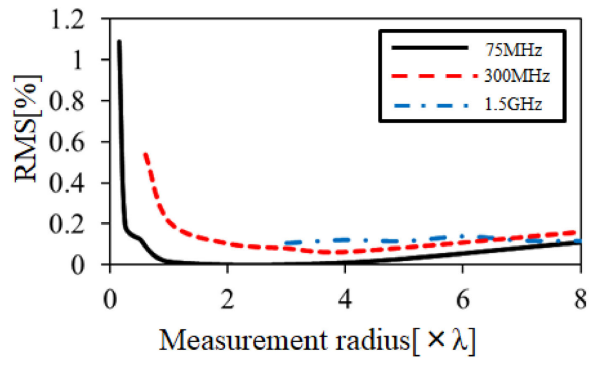

(c) RMS for measurement radius

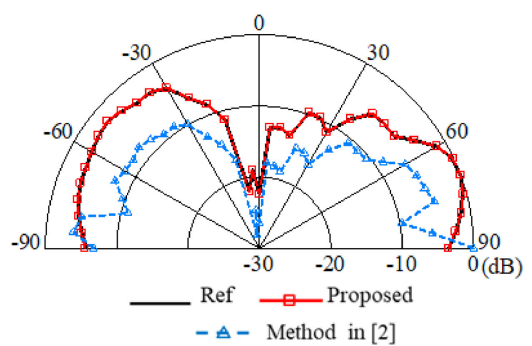

(b) Far field (yz)

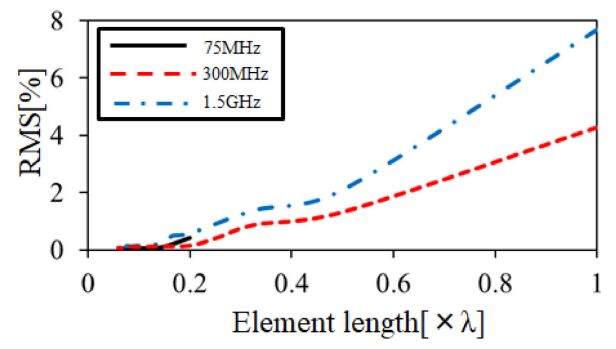

(d) RMS for element length

Fig. 2. Simulation results for semi-cylindrical model 
In addition, we will also consider the car model shown in Fig. 3(a). The size of the model is approximately $1 / 5$ of the actual car size. Fig. 3(c) shows a comparison of reference values and estimated values in the far field of $\mathrm{zx}$ and yz planes at 1.5 GHz. Near field measurement radius is $1 \mathrm{~m}$, and the element length of the linear approximation model is $0.1 \lambda$ or less. The relative residual at this time is $10^{-6}$, and the RMS is $0.296 \%$. Both main polarization and cross polarization can be correctly estimated in the zx and yz planes.

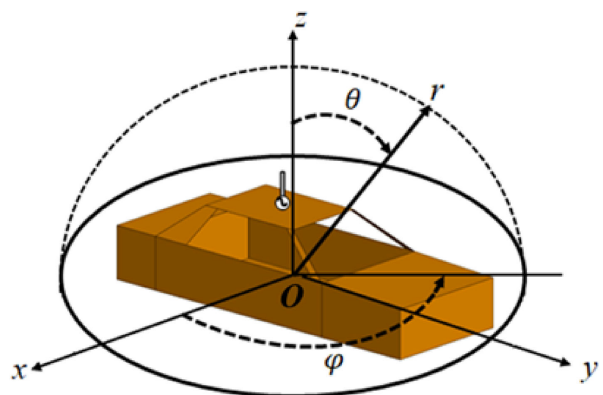

(a) Car model

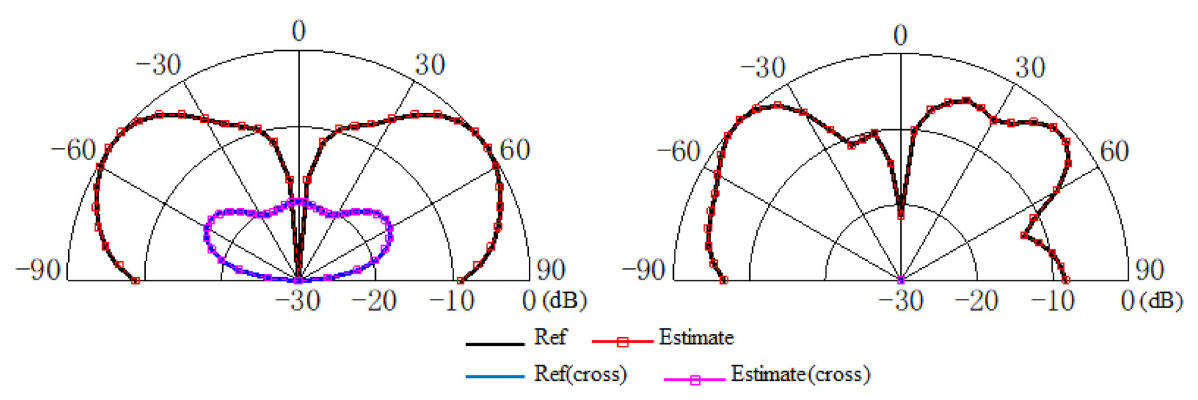

(c) Far field (zx/yz)

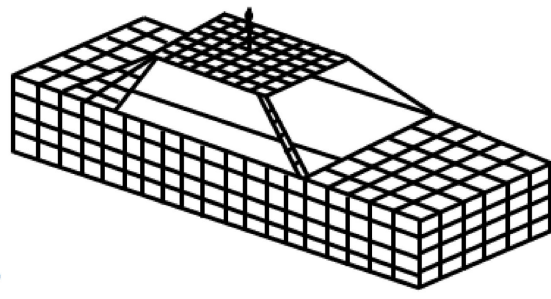

(b)Linear approximation

ults for car model

\section{Conclusion}

In this paper, the accuracy of far field estimation at high frequencies was improved by introducing a Hermitian transpose in an inverse problem defined by using hemispherical near field and a linear approximation model. In order to estimate accurately, the relative residual in the inverse problem needs to be about $10^{-6}$ or less. In addition, it was found that it is necessary to set the measurement radius to $1 \lambda$ or more and to set the linear element length of the linear approximate model to $0.2 \lambda$ or less for accurate estimation. Examination in noise consideration and measurement are left for future work. 